\title{
Hushing mTOR boosts immunity to pathogens
}

The serine/threonine protein kinase mTOR (mammalian target of rapamycin) senses and integrates diverse environmental signals to regulate cell growth and metabolism. Two studies in Nature Immunology now show that inhibiting the activity of mTOR can promote protective immunity to pathogenic intracellular infections.

Ivanov and Roy set out to investigate how the immune system can distinguish between pathogenic and non-pathogenic strains of the same infection. The authors infected macrophages with pathogenic Legionella pneumophila or with a non-pathogenic mutant of this bacterium and compared post-translational modifications of proteins in each group. They found that phosphoinositide 3-kinase (PI3K) and AKT, which activate mTOR, as well as mTOR itself, were ubiquitylated in macrophages infected with pathogenic L. pneumophila, but were phosphorylated in macrophages infected with the mutant strain. This suggests that the recognition of pathogenic, but not of



non-pathogenic, bacteria suppresses mTOR signalling in macrophages by targeting components of this pathway for proteasomal degradation.

Suppression of mTOR activity in L. pneumophila-infected macrophages increased their production of pro-inflammatory cytokines, including interleukin-6 (IL-6) and IL- $1 \beta$, and decreased their production of anti-inflammatory cytokines. The authors found that mTOR blockade biased cytokine production patterns in macrophages by inhibiting cap-dependent mRNA translation - a process that $\mathrm{mTOR}$ promotes by driving assembly of the eukaryotic translation initiation factor 4F (eIF4F) complex. Blocking cap-dependent translation favoured the translation of pro-inflammatory cytokine transcripts, as these are more abundant in macrophages than those that encode anti-inflammatory cytokines. Importantly, the suppression of mTOR enhanced protective immunity to L. pneumophila, as mice with a myeloid cell-specific deletion of mTOR had reduced bacterial loads following L. pneumophila infection, whereas mice in which $\mathrm{mTOR}$ signalling was constitutively activated showed impaired clearance of the bacteria.

The study by Keating et al. examined the role of mTOR in immunity to influenza virus. Their interest in mTOR stemmed from earlier work, which showed that rapamycin-mediated inhibition of mTOR promotes the development of memory $\mathrm{CD}^{+} \mathrm{T}$ cell responses. The authors tested whether rapamycin treatment during immunization with a non-infectious H3N2 influenza virus strain promotes protective immunity to subsequent infection with a lethal heterotypic influenza virus strain $(\Delta$ Vn1203). Compared with controls, mice who received rapamycin showed increased survival, experienced less weight loss and cleared the $\Delta \mathrm{Vn} 1203$ virus more rapidly. Rapamycin also enhanced protective immunity when mice were subjected to other normally lethal subtypes of influenza virus following prior immunization with the $\mathrm{H} 3 \mathrm{~N} 2$ strain.

Strikingly, although rapamycin increased the number of virusspecific memory $\mathrm{CD} 8^{+} \mathrm{T}$ cells, these cells were not responsible for the improved cross-protective immunity to influenza virus. Instead, rapamycinmediated protection was dependent on $\mathrm{CD}^{+}{ }^{+} \mathrm{T}$ cells and $\mathrm{B}$ cells. The authors found that the induction of activation-induced cytidine deaminase (AID) in B cells was dependent on signalling via mTOR complex 1 , and that rapamycin decreased germinal centre formation and classswitching responses in B cells during primary influenza virus infection. This limited the generation of IgG antibodies that were highly specific for immunodominant epitopes of haemagglutinin on the $\mathrm{H} 3 \mathrm{~N} 2$ strain, and increased the levels of IgM antibodies that were specific for more conserved haemagglutinin epitopes. So, mTOR blockade seems to promote crossprotective immunity to influenza virus by shaping an antibody repertoire that is less focused on a single strain of influenza virus and better able to target multiple variants of the virus.

ORIGINAL RESEARCH PAPERS Ivanov, S. S. \&

Roy, C. R. Pathogen signatures activate a ubiquitination pathway that modulates the function of the metabolic checkpoint kinase mTOR. Nature Immunol. http://dx.doi. org/10.1038/ni.2740 (2013) | Keating, R. et al. The kinase mTOR modulates the antibody response to provide cross-protective immunity to lethal infection with influenza virus. Nature Immunol. http://dx.doi.org/10.1038/ni.2741 (2013) 